\title{
Espacio-interfaz del Foro de Turismo Sustentable en territorios de gobernabilidad difusa
}

\author{
Interface-space of the forum for sustainable tourism in territories of diffuse governability \\ - María Elena Tosello \\ Universidad Nacional del Litoral, Argentina \\ metosello@fadu.unl.edu.ar \\ - Enrique Raúl Mihura \\ Universidad Nacional del Litoral, Argentina \\ emihura@fich.unl.edu.ar \\ - Patricia Beatriz Mines \\ Universidad Nacional del Litoral, Argentina \\ pmines@fadu.unl.edu.ar \\ María Georgina Bredanini Colombo \\ Universidad Nacional del Litoral, Argentina \\ gbredani@fadu.unl.edu.ar
}

\begin{abstract}
The interface-space of the forum for sustainable tourism constitutes a mediation threshold intended to exchanges between different semiotic systems, which stimulate dynamic processes of interaction, dialogue y negotiation in sociotechnical networks. The basis, methodological procedures and main results of the project are exposed, as well as the design and evaluation principles for habitable interface-spaces that guide the proposals. Finally, the paper outlines relevant actions for addressing collaborative construction processes related to social, communicative, technical and institutional dimensions.
\end{abstract}

Keywords: Interface-Space, Sustainable Tourism, Collaborative Construction, Interaction Processes, Governance Systems

\section{Introducción}

El territorio conformado por las áreas costeras de las localidades de Santa Fe, San José del Rincón y Arroyo Leyes, ha sido definido como una "micro-región insular" en proyectos de investigación en curso. Este ambiente se caracteriza por la presencia de riachos, arroyos e islas que poseen alta biodiversidad; con un patrimonio natural $\mathrm{y}$ cultural asociado a esa riqueza y a las huellas de los usos y procesos productivos que en ellos tienen y tuvieron lugar; con poblaciones y dinámicas heterogéneas; servicios e infraestructuras insuficientes; patrones de uso del suelo no concertados interjurisdiccionalmente; y diversos grados de concientización y vulnerabilidad frente al riesgo hídrico.

Los procesos interdependientes que allí se despliegan, no se diagnostican adecuadamente $y$ el paisaje se modifica con imprudencia, provocando un deterioro creciente del área por el desarrollo insustentable y el desaprovechamiento de los recursos; la ausencia de políticas públicas, procedimientos de gestión y legislaciones apropiadas; la fragmentación informacional, territorial y política; y la falta de documentación consistente y accesible, y de espacios de diálogo y medios de articulación para la definición de planes estratégicos integrados (incluido el turismo) entre entidades gubernamentales, no gubernamentales, privadas y pobladores.

El turismo ha experimentado un gran crecimiento en la micro-región insular, sin embargo no logra desarrollar aún su potencial transformador y transversal. La ausencia de una planificación conjunta del crecimiento de esta actividad, podría poner en riesgo no sólo los negocios vinculados al turismo, sino la calidad de vida de los pobladores.

Para poner en acto procesos dialógicos plurales, es imprescindible compartir la información y disponer de una comunicación fluida entre todos los sectores involucrados. Los entornos virtuales interconectados pueden contribuir al desarrollo sustentable de este territorio, a través de espacios virtuales interactivos o espacios-interfaz que brinden acceso a información calificada vinculada al turismo, y faciliten la comunicación entre instituciones públicas y empresas privadas, intermediarios turísticos y usuarios, considerando la diversidad de expectativas de los diferentes actores sociales, e integrando capacidades y recursos (www.itcilo.org/delnet).

Si bien las TIC pueden colaborar en procesos colectivos de producción de sentido, potenciar las experiencias de los usuarios y mejorar la visibilidad de las producciones, la apropiación de los espacios mediatizados por instrumentos tecnológicos no está garantizada sólo por la disponibilidad de tecnologías. La falta de atención a principios de diseño visual y de interacción aumenta la incertidumbre de algunos sujetos que son incapaces de lograr un uso satisfactorio y efectivo de las TIC (San Martín, 2013), ocasionando que sus prácticas cotidianas se disocien de los dispositivos socio-técnicos.

Por estos motivos, nuestro proyecto de investigación 
propone consolidar el proceso de constitución de un foro de turismo sustentable para la micro-región insular Santa Fe, Rincón, Arroyo Leyes, mediante un espacio-interfaz inclusivo que articule los vínculos entre los sujetos y las producciones mediatizadas, posibilitando la recopilación, divulgación e intercambio de información relevante; la comunicación, el diálogo y la colaboración entre actores públicos y privados; y la promoción de planes y emprendimientos vinculados al turismo sustentable, para alcanzar un desarrollo territorial integral, equilibrado y consensuado.

\section{Procedimientos metodológicos}

El espacio-interfaz se considera integrado al hábitat humano, y como todolugar habitado, manifiesta las costumbres, valores y sentidos de un contexto histórico aumentado y simbiótico que promueve nuevos modos de habitar.

En el diseño y puesta en obra de espacios-interfaz habitables de construcción colectiva, confluyen múltiples procesos en los cuales intervienen una diversidad de elementos heterogéneos e interdependientes (sujetos, prácticas sociales, medios, informaciones, tecnologías, etc.), cuyas interrelaciones configuran un sistema complejo. El análisis y la intervención en este tipo de sistemas, requiere de un instrumento que permita interpretar el comportamiento del mismo como una totalidad organizada (García, 1994), por lo cual se abordará desde una perspectiva interdisciplinaria, sistémica y heurística.

La metodología de investigación proyectual de espaciosinterfaz habitables, permite analizar y evaluar en forma integral la complejidad de estos sistemas, enfocándose en las necesidades y condiciones de contextos y usuarios concretos, en este caso autoridades municipales y provinciales, pobladores e investigadores de la Universidad Nacional del Litoral, emprendedores privados y consumidores de turismo, para explorar alternativas creativas de solución a los problemas, integrando diversas miradas e interpretaciones en un ambiente abierto que fomente el diálogo y dé lugar a lo emergente, a través del enfoque multidimensional de la interdisciplina y el pensamiento proyectual.

Esta metodología, que utiliza técnicas de investigación cualitativa para realizar un proceso de reflexión en la acción (Schön, 1992), ofrece una matriz flexible estructurada en tres etapas: Planificación-Diseño; Construcción-Experimentación; y Evaluación-Ajustes, pudiéndose priorizar una instancia sobre otra de acuerdo a las necesidades, lenguajes y sentidos que vayan surgiendo en el transcurso del trabajo, por eso el orden de las actividades se establece a partir de interacciones entre los actores en reuniones de trabajo que tienen lugar en espacios físicos y en debates virtuales.

Cabe aclarar que la micro-región insular Santa Fe, Rincón, Arroyo Leyes es un área inundable de gobernabilidad difusa (Fig.1), cuyo sistema complejo es tomado como "sujeto del desarrollo", con capacidades suficientes para planificar y gestionar programas y proyectos que generen procesos de crecimiento económico con equidad y sustentabilidad ambiental, así como planes de contingencia frente a situaciones extremas.

La evaluación se enfoca en aspectos performativos, a fin de verificar el grado de efectividad de las propuestas de diseño y conocer si los espacios-interfaz responden o no a los requerimientos y propósitos planteados, y si se integran adecuadamente con la dinámica de los espacios físicos, aplicando una triangulación de técnicas: observación participante, entrevistas y encuestas. Pero antes de la evaluación a partir de las pruebas con usuarios, se deben efectuar varios procesos previos, interdependientes e iterativos: el análisis y diagnóstico del marco sociotécnico; el diseño y construcción de los espacios-interfaz donde tendrán lugar las experiencias e inscripciones; y la planificación de estrategias de acción pertinentes para abordar las prácticas con los grupos sociales participantes.

Cada etapa integra subprocesos con objetivos y actividades particulares interrelacionadas. El diseño de un nuevo sistema de gobernanza interjurisdiccional, basado en la asociación democrática entre sujetos, organizaciones sociales, gobiernos y empresas, es uno de los subprocesos incluidos en la etapa de Planificación.

Para la apropiación social de un instrumento tecnológico, la etapa de Planificación es un momento clave donde se identifican los requerimientos y el flujo de interacciones. En este subproceso se utiliza el método de análisis de usuarios, influencias e intereses presentado por Ballejos y Montagna (2008). En la etapa de Construcción, para el desarrollo del software se utiliza RUP (Jacobson, Booch, Rumbaugh, 2000), que se define en términos de metas estratégicas, actividades y artefactos, habilitando un proceso continuo, iterativo e incremental mediante pruebas y retroalimentación, que garantiza el cumplimiento de ciertos estándares de calidad.

En cuantoa la organización, recopilación y administración de los datos, dado que en este tipo de sistemas es necesario contar con información geográfica, se tiene en cuenta la IDE (Integrated Development Environment) de la Provincia, la cual se concibe como un Sistema de Información Geográfica implementado sobre la Red (López Otero, Luaces, Paramá, 2007), y los estándares para almacenamiento y acceso a información geográfica, de manera que la plataforma que se utilice sea interoperable con otros sistemas IDE. 


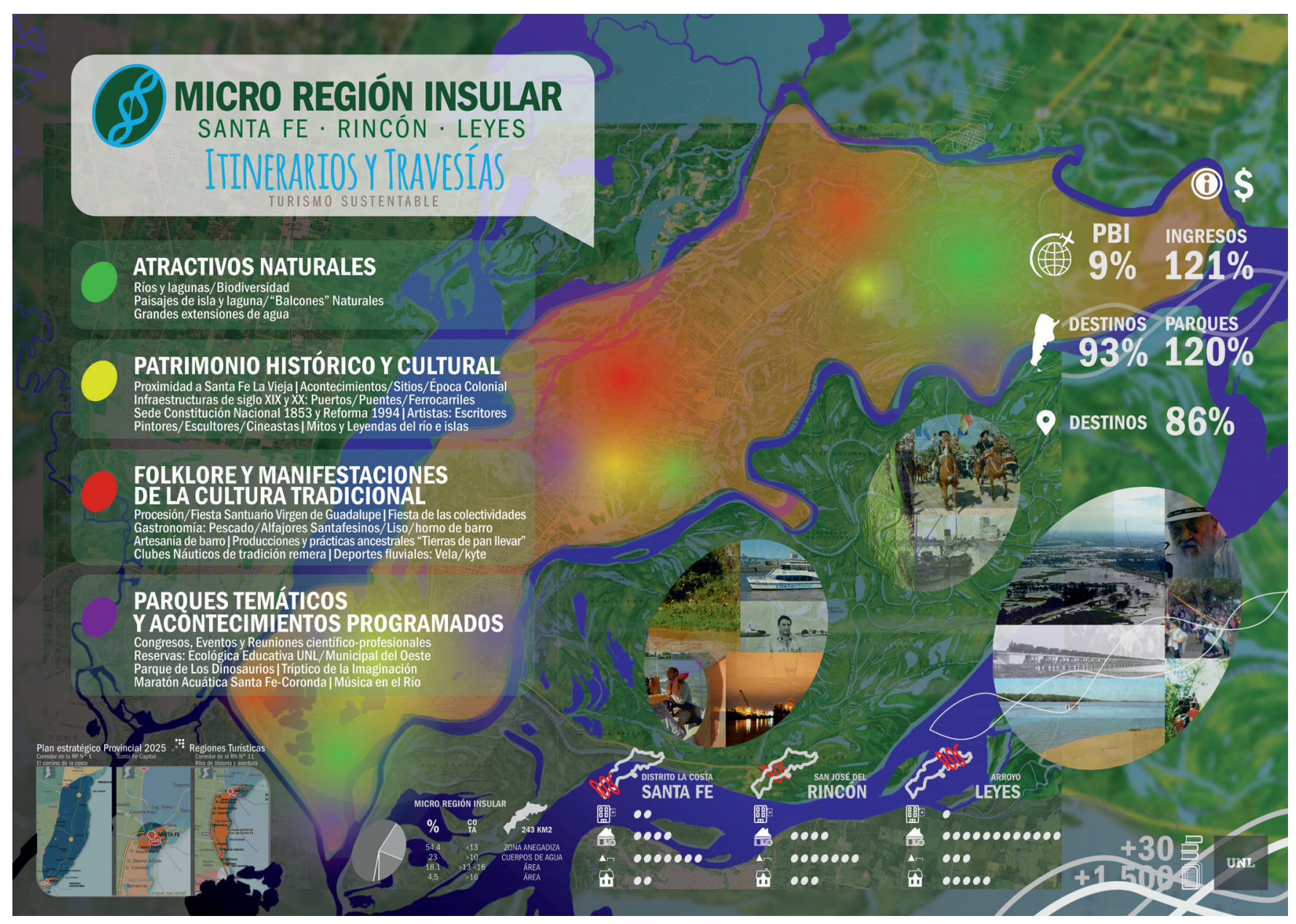

Figura 1: Micro-región insular Santa Fe, Rincón, Arroyo Leyes. Relevamiento de recursos turísticos e impacto (elaboración propia).

\section{Resultados}

El espacio-interfaz del Foro de Turismo Sustentable de la micro-región insular Santa Fe, Rincón y Arroyo Leyes, constituye un umbral de mediación destinado a intercambios entre distintos sistemas semióticos, integrados por sujetos, representaciones y discursos, que estimula procesos dinámicos de interacción, diálogo y negociación en redes socio-técnicas y posibilita:

- Reunir, catalogar y acceder a información consistente sobre turismo sustentable, en un repositorio multimedial abierto;

- Contribuir al desarrollo de conocimientos y capacidades para el diagnóstico, la previsión y la toma de decisiones consensuadas, favoreciendo planes y emprendimientos vinculados al turismo sustentable por parte de entidades públicas, privadas o mixtas;

- Impulsar procesos de construcción colaborativa de políticas públicas para la definición de condiciones de sustentabilidad, planes de contingencia y legislaciones apropiadas frente a situaciones extremas;

- Generar oportunidades de aprendizaje para la apropiación efectiva de las TIC por parte de los diversos actores, motivando la vivencia de experiencias significativas que faciliten las migraciones entre los espacios físicos y virtuales del Foro.

\section{Principios de diseño y evaluación}

Dado que el pensamiento proyectual ofrece un abordaje privilegiado en la creación y comprensión de los nuevos espacios para el ejercicio de la ciudadanía, se exponen los aspectos y factores a considerar en el análisis, diseño y evaluación de espacios-interfaz habitables (Tosello, 2015), fundados sobre principios éticos y sobre las condiciones políticas y organizacionales de un contexto situado (dimensión Institucional), que se agrupan según las áreas epistemológicas que se entrecruzan en la producción de narrativas hipermediales: las dimensiones Social, Tecnológica y Comunicacional o Textual.

Desde la dimensión Social se definen cuestiones prioritarias que orientan las decisiones de diseño: necesidades y requerimientos de los usuarios; perfil y rol actancial de los mismos (edad, nivel educativo, experiencia con TIC, preconceptos, etc.); contexto cultural y tecnológico donde se encuentran; naturaleza de los contenidos a alojar; valores ético-políticos a respetar; mecanismos de participación; 
estrategias de difusión, entrelazamiento y "acompañamiento" de los sujetos en su tránsito entre lo físico y lo virtual, para atravesar el umbral del espacio-interfaz en una atmósfera de confianza, ya que un buen diseño y un sistema tecnológico robusto no son condiciones suficientes para acreditar la apropiación social del espacio y la sostenibilidad.

En la dimensión Tecnológica se debe considerar: la configuración promedio de hardware de los usuarios (por ej.: el tamaño y resolución de las pantallas); la calidad del servicio web disponible (ancho de banda); los softwares de acceso abierto y los servicios web más utilizados por los usuarios; y en el caso de realizar desarrollos de programación originales, es esencial recurrir al lenguaje más conveniente, difundido y estable según el tipo de aplicación, evaluar los recursos humanos con que se cuenta, y explicar las funciones y el diagrama de flujos del programa a través de códigos comentados.

En la dimensión Comunicacional o Textual es preciso tener en cuenta una serie de normas lingüísticas (verbales y visuales), de usabilidad y de interacción. Tanto Nielsen, Tognazzini como Rodríguez Barros enuncian pautas de diseño en base a principios heurísticos de usabilidad. Mientras que Nielsen (1994b) propone diez principios básicos a considerar en el diseño de interfaces, Rodríguez Barros los complementa incorporando variables entrelazadas por el eje conductor del pensamiento proyectual, orientadas a lograr condiciones adecuadas de accesibilidad, experiencias gratificantes y modos originales de participación (Rodríguez Barros, 2008; 2012). Por su parte, Tognazzini plantea diecinueve principios generales enfocados específicamente al diseño de interacción (http://asktog.com/atc/principles-of-interaction-design/).

A las reglas de estos tres autores, Tosello (2015) incorpora nuevos aportes $\mathrm{y}$ formula un conjunto unificado de recomendaciones para guiar el diseño de espacios-interfaz habitables, que pueden aplicarse al desarrollo de hipermedios de autor (donde se interviene en todas las dimensiones), o a la adecuación socio-técnica de interfaces de sistemas colaborativos tales como Moodle, DSpace, etc. Estas pautas además, constituyen categorías de análisis y evaluación para diagnosticar la habitabilidad de todo tipo de sistema hipermedial, aplicación o sitio web. Si bien todos los principios están relacionados con la interacción y la usabilidad, los mismos se clasifican en:

- Factores generales vinculados al sistema: Visibilidad del estado del sistema; Funcionalidad; Contenido; Herramientas Colaborativas; Ayuda y documentación; y Accesibilidad.

- Aspectos relacionados a la interfaz gráfica: Estética; Identidad; Color; Legibilidad; Consistencia; y Uso de metáforas.

- Aspectos específicos de interacción: Uso de objetos estándar; Utilizar el lenguaje de los usuarios; Simplicidad; Facilidad de aprendizaje; Eficiencia del usuario; Estado y seguimiento de las acciones; Ley de Fitts; Reducción de latencia; Protección del trabajo de los usuarios; Prevención de errores; y Reconocimiento, diagnóstico y recuperación de errores.

- Factores de navegación y orientación espacial: Navegación orientada y visible; Interfaces explorables; y Encontrabilidad o rastreabilidad.

\section{Discusión}

Para finalizar, se enuncian las acciones que se consideran relevantes al abordar procesos de construcción colaborativa de espacios-interfaz para redes socio-técnicas, en relación a las dimensiones antes citadas (Fig. 2).

\section{Dimensión Social}

Impulsar procesos colaborativos y dialógicos dinámicos, que den lugar a la generación de conocimiento abierto, con estrategias de presencia sostenida para promover la inclusión, favoreciendo la migración y las experiencias significativas. Seleccionar temáticas convocantes que sean de interés para los participantes, a partir de problemáticas detectadas. Fomentar la gestión plural para consensuar estrategias de participación mediatizada responsable.

\section{Dimensión Textual}

Diseñar espacios multidimensionales, adaptables a diversas necesidades y contextos; fáciles de usar, abiertos a aportes, trayectos, prácticas e inscripciones múltiples; con ámbitos flexibles y personalizables. Facilitar la orientación espacial, la circulación y las búsquedas semánticas; y potenciar la producción y el intercambio multidireccional de diversos tipos de signos y sus relaciones, que se visibilicen en simultáneo y en tiempo real.

\section{Dimensión Tecnológica}

Integrar e interconectar bases de datos, sistemas de gestión, redes sociales y diversos servicios web de AA. Utilizar lenguajes de programación de código abierto y motores de bases de datos difundidos, sólidos, livianos, de fácil instalación y con soporte técnico del proveedor y de foros de usuarios, que sean funcionales desde el prototipo. Investigar e incorporar desarrollos existentes para resignificarlos y reutilizarlos desde el marco sociotécnico.

\section{Dimensión Institucional}

Desarrollar e implementar estrategias de sostenibilidad viables que incluyan la administración y el mantenimiento, considerando variables económicas, tecnológicas y humanas. Promover políticas de Acceso Abierto a la información, el conocimiento y la educación, y dinámicas de participación activa y responsable. 


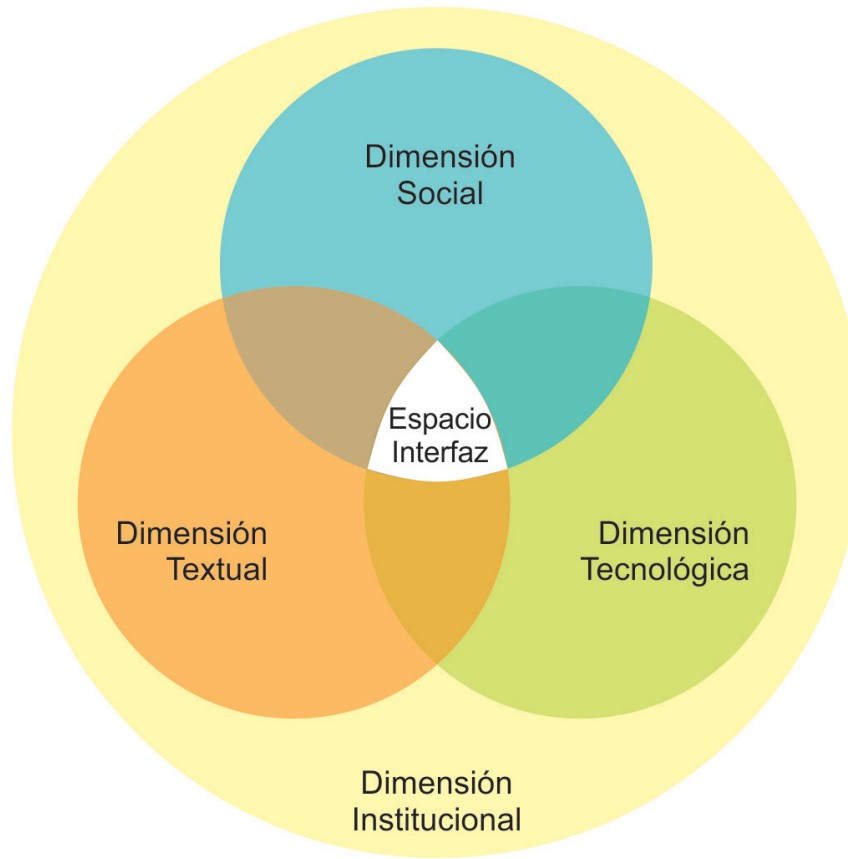

Figura 2: Diagrama de las dimensiones del espacio-interfaz.

\section{Conclusiones}

El principal aporte de esta iniciativa es la participación de un colectivo en el diseño y puesta en obra de espacios-interfaz habitables, y el desarrollo de procesos de aprendizaje amplios originados a partir de la temática movilizadora del turismo sustentable en un espacio simbiótico, dialógico y cooperativo, orientado a la concertación de estrategias para la construcción de lo público más allá del grado de mediatización y en base a características locales -saberes, recursos, requerimientos y condiciones históricas, sociales y culturales-, que se recuperan tanto desde el trabajo de campo como desde el paisaje donde se despliega la propuesta.

Para la gestión y sostenimiento del proyecto es fundamental la participación de todos los actores sociales en los debates, decisiones y acuerdos que se vayan planteando al interior del Foro, que busca provocar procesos transversales y dinámicos entre la comunidad, los gobiernos municipal y provincial, las empresas y asociaciones privadas, y la Universidad, la cual es responsable de asesorar, acompañar y brindar los instrumentos tecnológicos que posibilitan dichos procesos.

Actualmente el Foro se encuentra en proceso de constitución. Luego de su puesta en funcionamiento, se transferirá a un ente interjurisdiccional que adoptará y sostendrá tanto sus espacios físicos como los virtuales, definiendo estrategias de desarrollo en torno al turismo sustentable para impulsar prácticas de gobernanza innovadoras.

\section{Agradecimientos}

A la Secretarías de Ciencia y Técnica y de Relaciones Internacionales de la Universidad Nacional del Litoral por subsidiar las investigaciones y la participación en el congreso.

\section{Referencias}

Ballejos L., Montagna, M. (2008). Method for stakeholder identification in interorganizational environments. En Requirements Eng. Vol 13 pp. 281-297. DOI 10.1007/s00766008-0069-1. Springer-Verlag.

Centro internacional de formación de la Organización Internacional del trabajo. Curso de Turismo Sostenible y Desarrollo Local. Recuperado de www.itcilo.org/delnet García, R. (1994). Interdisciplinariedad y Sistemas Complejos. En: Leff, Enrique (comp.), Ciencias Sociales y Formación Ambiental. Barcelona: Ed. Gedisa.

Jacobson, I., Booch, G., Rumbaugh, J. (2000). El proceso unificado de desarrollo de software. Addison Wesley. López Otero, M., Luaces, M., Paramá, J. (2007). Implementación de un Servicio de Nomenclátor según la norma MNE y el estándar WFS-G. En IV Jornadas Técnicas de la IDE deEspaña, JIDEE 2007. España. Recuperado de http://www.idee.es/ resources/presentaciones/JIDEE07/ARTICULOS_JIDEE2007/ articulo3.pdf

Mihura, E (2009) Reflexiones y aportes para la sustentabilidad de procesos de gestión alternativa de la educación superior: estrategias para una educación para el desarrollo sustentable, Tesis de Maestria en Desarrollo Sustentable. FLACAM / UNL. Nielsen, J. (1994b). Heuristic evaluation. En Nielsen, J., and Mack, R.L. (Eds.), Usability Inspection Methods, New York: John Wiley \& Sons. Recuperado de http://www.nngroup. com/articles/ten-usability-heuristics/

Rodríguez Barros, D. (2012). Habitando los límites virtuales. Tesis doctoral. Universidad de Buenos Aires.

San Martín, P. (2013). Aspectos sociales y tecnológicos del Dispositivo Hipermedial Dinámico desarrollados en diferentes contextos educativos. En: Revista de Educación, Año 4, N 5, 81-98.

Schön, D. (1992). La formación de profesionales reflexivos. Hacia un nuevo diseño de la enseñanza y el aprendizaje en las profesiones. Barcelona: Paidós.

Tosello, M. E. (2015). El espacio-interfaz del dispositivo hipermedial dinámico. Tesis doctoral presentada en la Universidad Nacional de Rosario. 